Research Article

\title{
Delayed Feedback Control of Bao Chaotic System Based on Hopf Bifurcation Analysis
}

\author{
Farhad Khellat* \\ Department of Mathematics, Faculty of Mathematical Sciences, Shahid Beheshti University, Evin, Tehran, Iran.
}

Received 23 September 2014; Revised 24 October 2014; Accepted 25 November 2014

\begin{abstract}
This paper is concerned with bifurcation and chaos control in a new chaotic system recently introduced by Bao et al [9]. First a condition that the system has a Hopf bifurcation is derived. Then by applying delayed feedback controller, the chaotic system is forced to have a stable periodic orbit extracting from chaotic attractor. This is done by making Hopf bifurcation value of the open loop and the closed loop systems identical. Also by suitable tuning of the controller parameters, unstable equilibrium points become stable. Numerical simulations verify the results.
\end{abstract}

Keywords: Bao chaotic system, chaos control, delayed feedback, Hopf bifurcation.

\section{Introduction}

After three decades, chaos control remains an interesting and applied field in nonlinear control literatures yet; see for example [1,2]. Delayed feedback control (DFC) [3] is a simple tool to stabilize Unstable Periodic Orbits (UPOs) embedded in the strange attractors of chaotic systems. The method is reference free; it makes use of a control signal obtained from the difference between the current state of the system and the state of the system delayed by one period of the UPO. Successful implementation of this algorithm has been attained in diverse experimental systems [4] and references therein.

The task of stability analysis of DFC is not easy. Nevertheless, a full analytical eigenmode expansion of the linear delayed systems and a weakly nonlinear analysis has been given in [5]. In [6,7] local Hopf bifurcation of scalar delayed model and its control under DFC is considered. Authors in [8] have chosen feedback gain by trial and error and delay is tuned by the energy of input signal obtained in simulations. Recently, similar methods have been used in $[9,10]$ to tune DFC parameters to control of chaos.

In 2008, a new system was introduced which could be displayed one-scroll and double-scroll chaotic attractors with only two equilibrium points and can be found to be robust chaotic in a very wide parameter domain [11]. In this paper we analyze this system for finding Hopf bifurcation values. Then we apply DFC to this model. The controller parameters are determined to stabilize UPOs. To tune the controller parameters delay and gain, we use this fact that the period of periodic orbits (stable or unstable) born at bifurcation values,

* E-mail address: f-khellat@sbu.ac.ir ISSN: 1791-2377 @ 2015 Kavala Institute of Technology. All rights reserved. depends on the roots of characteristic equation at bifurcation points. Consequently delay is tuned such that the root of characteristic equation of the closed loop system becomes the root of characteristic equation of the open loop system. Since the stability of periodic solution born from this Hopf bifurcation, is dependent on the gain of the controller, by a suitable choice of gain, the UPO may become stable.

The rest of the paper is organized as follows. In Section 2 , the robust chaotic system is revisited and Hopf bifurcation of this system is analyzed. In Section 3, the closed loop system under DFC is studied and the controller parameters are determined based on Hopf bifurcation. The results of simulations are presented in section 4 to verify the effectiveness of method to tune the parameters of DFC. The conclusion is given in section 5 .

\section{Revision of the System and Hopf Bifurcation Analysis}

In this section the chaotic system, equilibrium points and the characteristic equation are given from [11]. Then we study the Hopf bifurcation of the system analytically. Consider the following chaotic system:

$$
\left\{\begin{array}{l}
\dot{x}=y-z \\
\dot{y}=x z+c \\
\dot{z}=\mu-\exp (x y)
\end{array}\right.
$$

where $\mu$ and $c$ are parameters of the system. In Fig. 1 the chaotic attractor in the phase space $(x, y, z)$ has been shown for $c=0$ and $\mu=5$. 


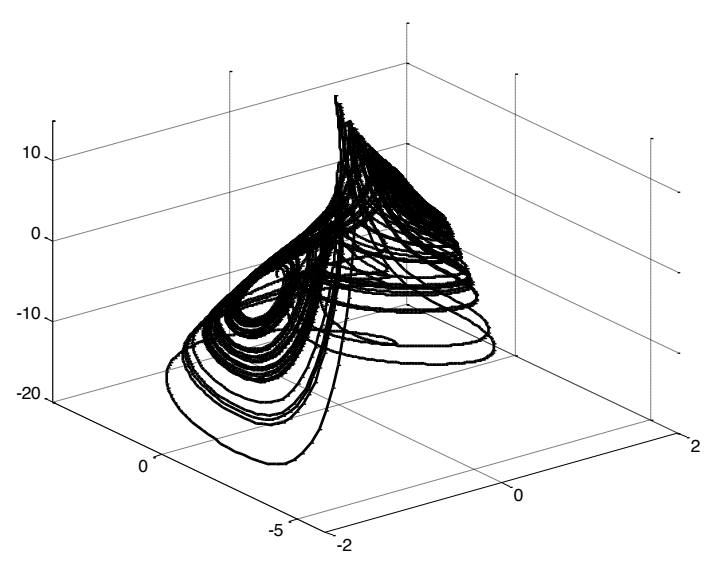

Fig. 1. Chaotic attractor of (1) for $c=0$ and $\mu=5$.

For $\mu>1$, the equilibrium points of the system are

$$
S_{1,2}=( \pm \sqrt{\ln \mu}, \pm \sqrt{\ln \mu}, \mathrm{mc} / \sqrt{\ln \mu})
$$

and the characteristic equations at equilibrium points are

$$
\Delta(\lambda)=\lambda^{3}+\lambda^{2}+\left(\mu \ln \mu \pm \frac{c}{\sqrt{\ln \mu}}\right) \lambda+2 \mu \ln \mu=0
$$

Let $c$ be the bifurcation parameter and $\mu$ be constant. To find values of $c$ at which Hopf bifurcations are occurred, let $\lambda=\mathrm{i} \omega_{0}$ be a root of (3), then

$\Delta\left(\mathrm{i} \omega_{0}\right)=-\mathrm{i} \omega_{0}^{3}-\omega_{0}^{2}+\mathrm{i}\left(\mu \ln \mu \pm \frac{c}{\sqrt{\ln \mu}}\right) \omega_{0}^{2},+2 \mu \ln \mu=0$.

Separating the real and imaginary parts, we have

$$
\left\{\begin{array}{l}
\omega_{0}=\sqrt{2 \mu \ln \mu} \\
\omega_{0}\left(\omega_{0}^{2}-(\mu \sqrt{\ln \mu} \pm c / \sqrt{\ln \mu})\right)=0
\end{array}\right.
$$

which implies that $c_{1,2}{ }^{*}= \pm \mu(\ln \mu)^{\frac{3}{2}}$ are candidates for Hopf bifurcation at bifurcation points $S_{1,2}{ }^{*}=( \pm \sqrt{\ln \mu}, \pm \sqrt{\ln \mu}, \mp \mu \sqrt{\ln \mu})$

Note that $S_{1,2}{ }^{*}$ are symmetric with respect to the $z$ axis [11]. First we study the behaviour of the system around $S_{1}{ }^{*}$. The other root of (3) is $\lambda=-1$. We proceed to calculate the real part of $\frac{d \lambda}{d c}$ i.e. $\frac{d}{d c}(\operatorname{Re}(\lambda))$, evaluated at $c=c_{1}^{*}$ as mentioned in [12]. By differentiating (3) implicitly with respect to $c$ and then simplify, we obtain

$$
\left.\frac{d}{d c}(\operatorname{Re}(\lambda))\right|_{c=c_{1}}=\frac{-1}{2 \sqrt{\ln \mu}(1+2 \mu \ln \mu)}
$$

Hence, the eigenvalues cross from the right half-plane (RHP) to the left half-plane (LHP) as $c$ is increased. $S_{1}^{*}$ is unstable for $c<c_{1}^{*}$ and is asymptotically stable for $c>c_{1}^{*}$. Similarly, The bifurcation point $S_{2}{ }^{*}$ is asymptotically stable for $c<c_{2}{ }^{*}$ and is unstable for $c>c_{2}{ }^{*}$. In Fig. 2 bifurcation diagram for $x$ versus $c$ is shown for $\mu=2.5$ and bifurcation values $c_{1,2}{ }^{*} \approx \pm 2.2$.

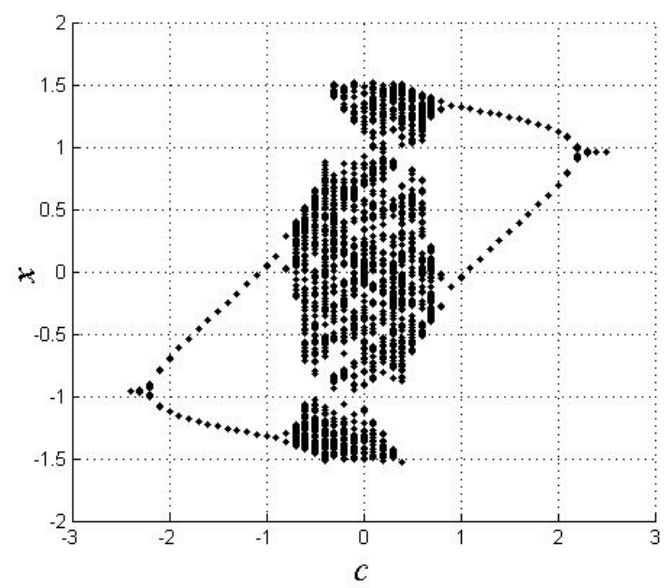

Fig. 2. Bifurcation diagram $x$ versus $c$ for $\mu=2.5, c_{1,2}{ }^{*} \approx \pm 2.2$.

\section{DFC Based on Hopf Bifurcation}

In order to improve the performance of a dynamic system or to avoid the chaotic phenomena, we need to control chaotic system to a regular target trajectory embedded in the chaotic attractor which is beneficial for working with a particular condition. In this paper we focus on DFC with the following control law:

$$
u(t)=k(s(t-T)-s(t))
$$

where $s(t)$ could be a linear or nonlinear function of the states variables and control input $u(t)$ could be applied to each of the system equations. For system (1) we assume that $s(t)=y(t)$ and the dynamic of $y(t)$ is affected by $u(t)$. So the closed loop model is obtained as:

$\left\{\begin{array}{l}\dot{x}=y-z \\ \dot{y}=x z+c+k(y(t-T)-y(t) \\ \dot{z}=-\exp (x y)\end{array}\right.$

Now we tune the parameters of the controller: feedback gain $k$ and delay time $T$.

\subsection{Stabilizing UPO}

To achieve the structure of unstable periodic orbit embedded in chaotic attractor, $T$ must be set as the period of that orbit. From Hopf bifurcation theory [10], we know that the period of orbits near the Hopf bifurcation point is approximately $2 \pi / \omega_{0}$. So we set $T=2 \pi / \omega_{0}$. Now $k$ must be determined such that after a Hopf bifurcation, period doubling routes to chaos is not occurred. To do this, we linearize the closed loop system around the equilibrium points.

The characteristic equation at $S_{1,2}{ }^{*}$ is

$$
\begin{aligned}
\Delta(\lambda)=\lambda^{3} & +(1+k(1-\exp (-T \lambda))) \lambda^{2} \\
& +\left(\mu \ln \mu \pm \frac{c}{\sqrt{\ln \mu}}+k(1-\exp (-T \lambda))\right) \lambda+2 \mu \ln \mu=0
\end{aligned}
$$


For $\lambda=i \omega_{0}$ and $T=2 \pi / \omega_{0}$, equations (3) and (8) are identical. Hence, similar to the open loop case, Hopf bifurcations are occurred for the closed loop system (7) at $c_{1,2}{ }^{*}= \pm \mu(\ln \mu)^{\frac{3}{2}}$ for all $k$. From Fig. 2, one can find that for some $c \in\left(c_{2}{ }^{*}, c_{1}{ }^{*}\right)$, chaos is appeared. To stabilize chaos, we have to move the roots of characteristic equation to the left by choosing a suitable $k$.

Since $k$ may be determined in terms of $\mu$ and $c$, to characterize the effect of $k$ on the stability of periodic orbits, we study the variations $\frac{d}{d c}(\operatorname{Re}(\lambda))$ and $\frac{d}{d \mu}(\operatorname{Re}(\lambda))$ at Hopf bifurcation values $c_{1,2}{ }^{*}$.

In Fig. 3(a), $\frac{d}{d c}(\operatorname{Re}(\lambda))$ is plotted versus $(k, \mu)$. It is shown that near Hopf bifurcation values $\operatorname{Re}(\lambda)$ is not changed with respect to the parameter $c$ for all values of $k$ and $\mu$ in an interval. In Fig. 3(b), $\frac{d}{d \mu}(\operatorname{Re}(\lambda))$ is plotted versus $(k, \mu)$. It shows that for some values of $k$ and $\mu$ in a region $\operatorname{Re}(\lambda)$ is increasing and $\frac{d}{d \mu}(\operatorname{Re}(\lambda))>0$ is decreasing in another region. To see the regions, the contour $\frac{d}{d \mu}(\operatorname{Re}(\lambda))=0$ of Fig. 3(b) is shown in Fig. 3(c). It shows that for values of $k$ and $\mu$ in gray region, the roots of the characteristic polynomial (8) lie on RHP and they increase with increasing $\mu$. In this case Hopf bifurcation is occurred. To achieve a period one orbit, the real part of the rightmost root of characteristic polynomial (8) must be in RHP and near the imaginary axis. That is for a known $\mu$, the value of $k$ in the gray region must be near the boundary curve which separates the gray and white regions. So by decreasing $k$ for a fixed $\mu$, periodic orbits with higher periods are obtained. For values of $k$ and $\mu$ on the boundary curve between gray and white region, the roots lie on the imaginary axis and for those values of $k$ and $\mu$ in the white region the roots are on LHP, i.e., where $\frac{d}{d \mu}(\operatorname{Re}(\lambda))<0$.

\subsection{Stabilizing Unstable Equilibrium Points}

Those values of $k$ for which $\frac{d}{d \mu}(\operatorname{Re}(\lambda))<0$, are suitable for stabilizing the equilibrium points. In this case the roots of characteristic equation are moved from RHP to LHP by increasing the parameters. From Fig. 3(c) it is clear that the condition $\frac{d}{d \mu}(\operatorname{Re}(\lambda))<0$ is held for large $\mu$ in a small interval and feedback gain $k$ is increased. To have an upper bound for $k$, we consider the effect of delay time $T$ on the stability of equilibrium points. Simply we find that $\frac{d}{d T}(\operatorname{Re}(\lambda))<0$ at bifurcation values. Therefore if $T$ is decreased, then the real part $\lambda$ is decreased and it implies the stability of equilibrium points. So for $T<2 \pi / \omega_{0}$ and suitable feedback gain $k$, equilibrium points become stable.

\section{Simulation Results}

In this section, we illustrate DFC with parameters that are adjusted by the method in Section 3, can stabilize many periodic solutions embedded in chaotic attractors. For all simulations we assume $c=0, \mu=5$ and the initial condition is $[1,-1,0.5]^{\mathrm{T}}$. The periodic orbits are plotted after transient time.

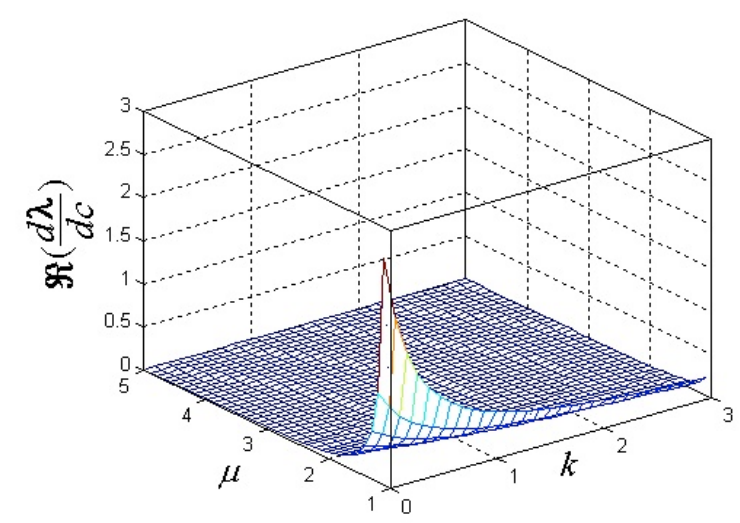

(a)

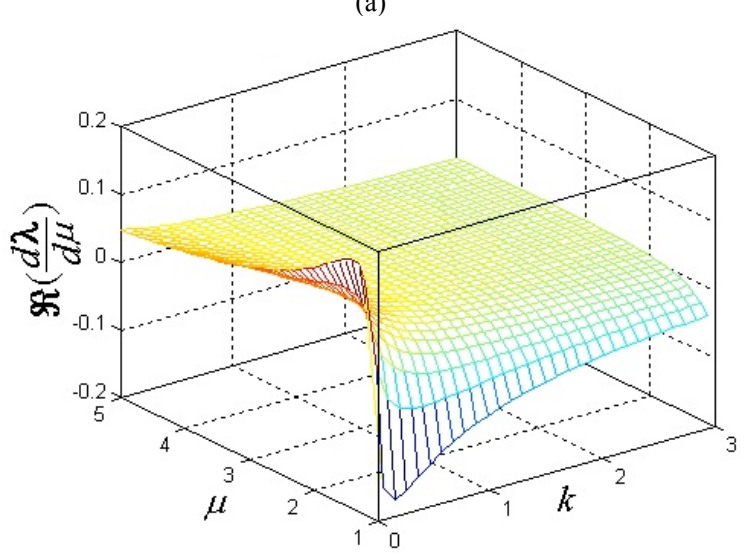

(b)

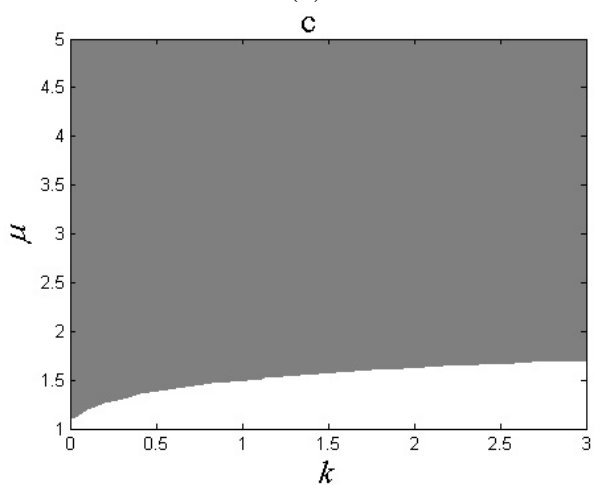

(c)

Fig. 3. The variations of (a) $\frac{d}{d c}(\operatorname{Re}(\lambda))$, (b) $\frac{d}{d \mu}(\operatorname{Re}(\lambda))$ and (c) the contour of $b$ where the gray regions indicates $\frac{d}{d \mu}(\operatorname{Re}(\lambda))>0$ versus $(k, \mu)$.

First from (4) we have $\omega_{0}=\sqrt{10 \ln 5} \approx 4.0118$, therefore we choose $T=2 \pi / \omega_{0} \approx 1.566$. We choose $k>1.18$, which implies that the roots of characteristic equation are in the

RHP and near the imaginary axis. The results of simulations are presented in Fig. 4 for some values of $k$. It is shown that for $k=1.7$, the periodic orbits which arise from a Hopf bifurcation become stable. By decreasing $k$ from $k<1.18$. periodic orbits with higher period embedded in the chaotic attractor become stable. 

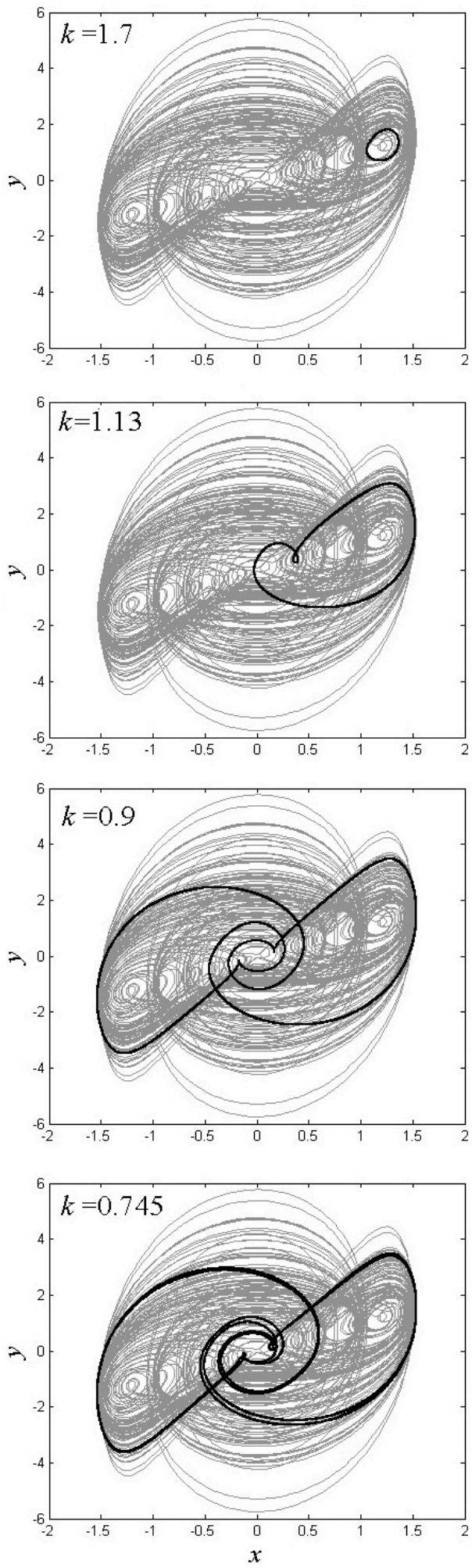

Fig. 4. Stabilizing periodic orbits by DFC for $T=1.566$ and for some values of feedback gain: $k=1.7$ (period one); $k=1.13$ (period two); $k=0.9$ (period four) and $\mathrm{k}=0.745$ (higher period).

Also, by the results obtained in the previous section, decreasing $T$ implies the characteristic eignvalues goes to LHP and the stability of equilibrium points is attained. This is shown in Fig. 5 for $T=1.466$ and $k=1.7$, then $S_{1}^{*}=[1.6094,1.6094,0]^{\mathrm{T}}$ is stabilized.
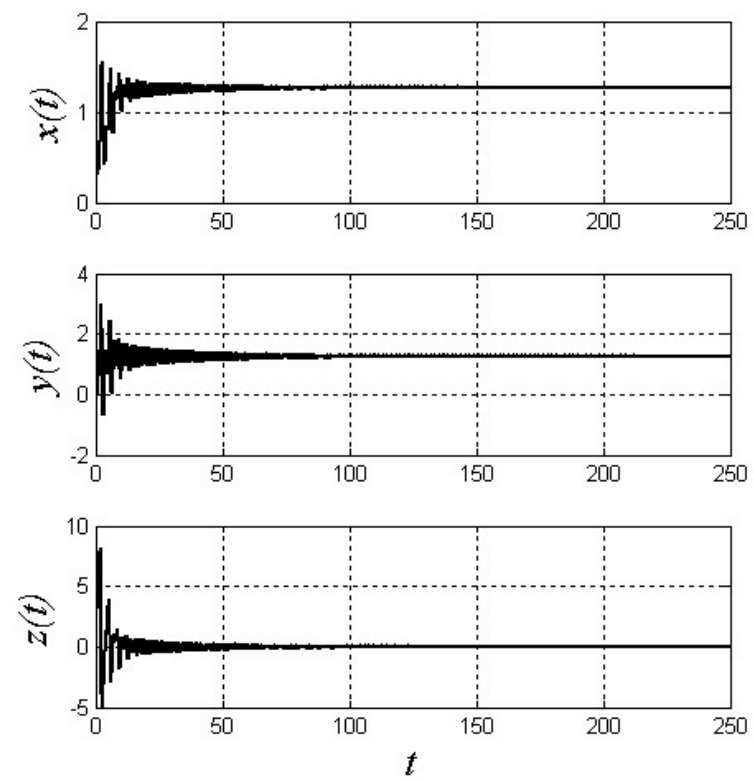

Fig. 5. Stabilizing equilibrium point $S^{*}{ }_{1}$ by DFC for $T=1.466$ and $k=1.7$.

In Fig. 6 bifurcation diagram of the closed loop system is plotted versus the parameter $c$. It shows that DFC is robust with respect to the variations of the parameter $c$, and period one orbit remains stable for a wide range of the parameter. It can be compared with the bifurcation diagram of the open loop system in Fig. 2.

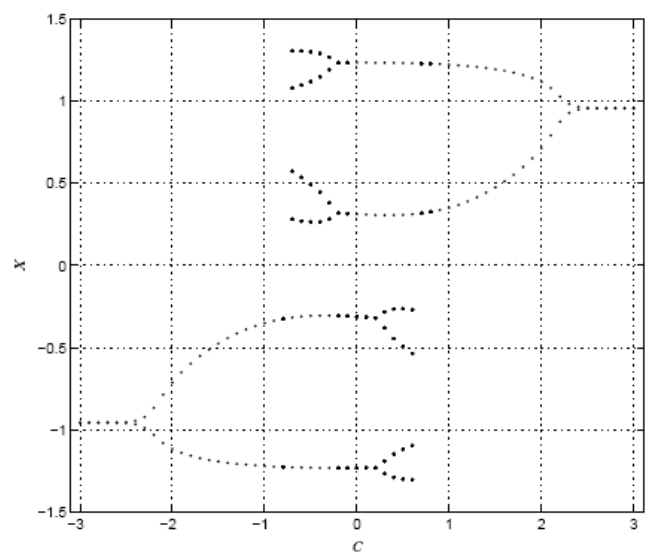

Fig. 6. Bifurcation diagram of the closed loop system for $\mu=2.5$, $T=2.9355$ and $k=1.7$.

\section{Conclusion}

In this paper first we studied Hopf bifurcation of a chaotic system. Points at which the system undergoes Hopf bifurcation are obtained. By analyzing the eigenvalues of the Jacobian matrix at bifurcation points, the stability of the equilibrium points and the periodic orbits were determined. Based on the results of Hopf bifurcation, DFC was investigated to stabilize UPOs and unstable equilibrium points of the chaotic system. Tuning the parameters containing feedback gain and delay time of the controller obtained. At last we showed the simulation results of DFC for some parameters. 


\section{Acknowledgment}

The author would like to thank Dr. Nastaran Vasegh for assistance in completing this work.

\section{References}

1. Y.N. Li, L. Chen, Z.S. Cai, and X.Z. Zhao, Study on chaos synchronization in the Belousov-Zhabotinsky chemical system, Chaos, Solitons and Fractals, vol. 17, pp. 699-707 (2003).

2. J. Sieber, E. Omelchenko, and M. Wolfrum, Controlling unstable chaos: Stabilizing chimera states by feedback, Physical Review Letters, vol. 112(5), pp. 054102 (2014).

3. J. Wang, X. Chen, and J. Fu, Adaptive finite-time control of chaos in permanent magnet synchronous motor with uncertain parameters, Nonlinear Dynamics, vol. 78(2), pp. 1321-1328 (2014).

4. K. Pyragas, Continuous control of chaos by self-controlling feedback, Physics Letters A, vol. 170(6), pp. 421-428 (1992).

5. E. Schöll and H.G. Schuster, (Eds.). Handbook of chaos control, John Wiley \& Sons, (2008).

6. A. Amann, E. Schöll, and W. Just, Some basic remarks on eigenmode expansions of time-delay dynamics, Physica A: Statistical Mechanics and its Applications, vol. 373, pp. 191-202 (2007).

7. N. Vasegh and A.K. Sedigh, Delayed feedback control of timedelayed chaotic systems: Analytical approach at Hopf bifurcation, Physics Letters A, vol. 372(31), pp. 5110-5114 (2008).
8. N. Vasegh and A.K. Sedigh, Chaos control via TDFC in timedelayed systems: The harmonic balance approach, Physics Letters A, vol. 373(3), pp. 354-358 (2009).

9. W. Shu-Hua, H. Jian-Hong, and X. Hai-Bo, Controlling chaos to unstable periodic orbits and equilibrium state solutions for the coupled dynamos system, Chinese Physics B, vol. 19(2), pp. 020509 (2010).

10. M.M. El-Dessoky, M.T. Yassen and S.E. Aly, Bifurcation analysis and chaos control in Shimizu-Morioka chaotic system with delayed feedback, Applied Mathematics and Computation, vol. 243, pp. 283-297 (2014).

11. R. Zhang, Bifurcation analysis for $\mathrm{T}$ system with delayed feedback and its application to control of chaos, Nonlinear Dynamics, vol. 72(3), pp. 629-641 (2013).

12. B. Bo-Cheng, L. Chun-Biao, X. Jian-Ping, and L. Zhong, New robust chaotic system with exponential quadratic term, Chinese Physics B, vol. 17(11), pp. 4022 (2008).

13. J. Guckenheimer and P. Holmes, Nonlinear oscillations, dynamical systems, and bifurcations of vector fields, vol. 42 Springer Verlag, New York (1983). 